\title{
(DES)PROTECCIÓN DE LOS DERECHOS DE LA INFANCIA EN EL CONTEXTO DE LA PANDEMIA
}

\author{
Patricia Muñoz, García
}




\title{
PATRICIA MUÑOZ GARCÍA
}

\begin{abstract}
Abogada de la Universidad Católica de Temuco. Magíster en Docencia Universitaria de la Universidad del Desarrollo. Entre los años 2004 y 2018 se desempeñó como fiscal adjunta especializada en derechos humanos, violencia de género y delitos sexuales, y como gerenta de la División de Atención a las Víctimas y Testigos. Fue la primera mujer presidenta de la Asociación de Fiscales en el Ministerio Público de Chile. En 2018 se convirtió en la primera defensora de la Niñez de Chile.
\end{abstract}




\section{(DES)PROTECCIÓN DE LOS DERECHOS DE LA INFANCIA EN EL CONTEXTO DE LA PANDEMIA}

El artículo $1^{\circ}$ de nuestra Constitución Política ${ }^{1}$ establece, para dar inicio a las bases de nuestra institucionalidad, que "Las personas nacen libres e iguales en dignidad y derechos”, y agrega, luego, que

El Estado está al servicio de la persona humana y su finalidad es promover el bien común, para lo cual debe contribuir a crear las condiciones sociales que permitan a todos y a cada uno de los integrantes de la comunidad nacional su mayor realización espiritual y material posible, con pleno respeto a los derechos y garantías que esta Constitución establece (Constitución Política de la República de Chile).

Al finalizar, añade que el Estado deberá “... asegurar el derecho de las personas a participar con igualdad de oportunidades en la vida nacional”.

Inicio este texto recordando este mandato constitucional porque, precisamente, a la luz de lo acontecido en Chile en la crisis social que se manifestó con mayor fuerza el 18 de octubre de 2019 y en la actual crisis sanitaria y de derechos humanos que representa la pandemia del Coronavirus, queda de manifiesto cómo aquellos mandatos establecidos para el Estado de Chile, independientemente de quién lidere su gobierno, siguen, en relación con los niños, niñas y adolescentes, sin cumplirse. Se trata de una situación que se evidencia con mayor claridad si a dichas exigencias constitucionales sumamos las obligaciones jurídicas adquiridas por el Estado el año 1990, cuando ratificó la Convención sobre los Derechos del Niño, tratado internacional que define y establece un paradigma desde el cual reconocer, tratar y proteger a niños, niñas y adolescentes por su condición de sujetos de derecho y no meros objetos de protección.

1. Constitución Política de la República, Chile, en línea en https://www.leychile.cl/ Navegar?idNorma $=242302$. 
Dicho tratado internacional establece, en su artículo $2^{\circ 2}$, que:

1. Los Estados Partes respetarán los derechos enunciados en la presente Convención y asegurarán su aplicación a cada niño sujeto a su jurisdicción, sin distinción alguna, independientemente de la raza, el color, el sexo, el idioma, la religión, la opinión política o de otra índole, el origen nacional, étnico o social, la posición económica, los impedimentos físicos, el nacimiento o cualquier otra condición del niño, de sus padres o de sus representantes legales. 2. Los Estados Partes tomarán todas las medidas apropiadas para garantizar que el niño se vea protegido contra toda forma de discriminación o castigo por causa de la condición, las actividades, las opiniones expresadas o las creencias de sus padres, o sus tutores o de sus familiares.

Vale la pena determinar, habida cuenta de lo que ha ocurrido en relación con la pandemia, cómo afecta e impacta la profunda inequidad y desigualdad que se vive en el país en los niños, niñas y adolescentes, y determinar si aquellas obligaciones centrales del Estado chileno han sido cumplidas por este o si, por el contrario, a pesar del desarrollo económico y social alcanzando en el país durante estos años, este no ha podido llegar, "sin distinción alguna", a todas y todos quienes habitan el país, en condiciones de igualdad y dignidad. Además de aquello, resulta necesario determinar si en el diseño e implementación de políticas públicas que se han desarrollado en el país, aquellas han sido capaces de ver y tratar a los niños, niñas y adolescentes como sujetos de derecho o si, más bien, siguen siendo tratados, tanto por los órganos del Estado como por la sociedad en su conjunto, como meros objetos de protección y de decisión de los adultos que les rodean.

Para analizar los puntos referidos, resulta necesario tener a la vista algunas cifras relacionadas con niños, niñas y adolescentes. Podemos observar que, al año $2017^{3}$, en Chile existían 4.259.155 niños, niñas y adolescentes. De este total, un 12,6 por ciento vive en la ruralidad y de 596.582 que se reconocen pertenecientes a un pueblo originario, 471.742 de ellas y ellos adscribe al pueblo mapuche. Un 5,1 por ciento de quienes se encuentran en situación de discapacidad tienen entre 0 y 5 años, un 5,8 por ciento tiene entre 6 y 13 años y un 6 por ciento entre 14 y 17 años. En relación con la situación de pobreza por ingresos, un 13,9 por ciento de los niños enfrenta

2. Convención sobre los Derechos del Niño, Naciones Unidas, en línea en https://www.leychile. $\mathrm{cl} /$ Navegar?idNorma $=15824$.

3. Defensoría de los Derechos de la Niñez, Informe Anual 2019, en línea en https://www. defensorianinez.cl/informe-anual/docs/II_cap0_2019_preambulo.pdf. 
esta dificultad en sus hogares, cifra que alcanza el 22,9 por ciento en el caso de la pobreza multidimensional. Del total de niños, niñas y adolescentes que viven en Chile, un 21,4 por ciento de quienes tienen entre 0 y 5 años, un 16,9 por ciento de quienes tienen entre 6 y 13 años y un 15 por ciento de quienes tienen entre 14 y 17 años ha presentado algún problema de salud, accidente o enfermedad, y de un total de 428.369 personas menores de 20 años que presentan trastornos de salud mental, 295.612 corresponden a niños y niñas entre los 0 y 14 años de edad. Finalmente, el Informe sobre Estadísticas Migratorias, elaborado en conjunto entre el Instituto Nacional de Estadísticas y el Departamento de Extranjería y Migración, estima que, al 31 de diciembre de 2019, en Chile vivían 209.866 niños, niñas y adolescentes migrantes ${ }^{4}$.

Considerando lo anterior, es evidente que las acciones estatales destinadas a proteger a los niños, niñas y adolescentes que viven en Chile deben estar diseñadas e implementadas integrando, como elemento central, la diversidad que representa la infancia y la adolescencia en nuestro país, a fin de entregar espacios efectivos en los que ellas y ellos puedan, además, y tal como lo mandata nuestra Carta Fundamental y lo refuerza la Convención sobre los Derechos del Niño, participar activamente, incidiendo en las definiciones de políticas o acciones públicas que les afectan o afectarán, toda vez que su invisibilización en la elaboración de estas hará imposible que les resulten atingentes y que sean capaces de responder, con eficiencia y oportunidad, a aquellas exigencias vitales que están teniendo hoy.

El Secretario General de Naciones Unidas ha enfatizado, con mucha claridad, que la pandemia del Covid-19 no solo es una crisis sanitaria, sino que también es una crisis de derechos humanos, $\mathrm{y}$ ha sostenido que:

La pandemia de Covid-19 es una emergencia de la salud pública, pero es mucho más. Es una crisis económica. Una crisis social. Y una crisis humana que se está convirtiendo rápidamente en una crisis de derechos humanos ${ }^{5}$.

Esta declaración es coherente con lo que ha acontecido en nuestro país, donde hemos debido enfrentar diversas y variadas restricciones a derechos fundamentales, por de pronto, la restricción absoluta o parcial de nuestra libertad de desplazamiento,

4. Instituto Nacional De Estadísticas (INE), Departamento De Extranjería y Migración (DEM), 2020, Estimación de personas extranjeras residentes habituales en Chile al 31 de diciembre de 2019.

5. Naciones Unidas, Ginebra, Declaración emitida el 23 de abril de 2020, "Miremos a través del prisma de los derechos humanos", en línea en https://www.un.org/es/coronavirus/articles/ miremos-prisma-derechos-humanos-respuesta-covid-19. 
y donde también hemos visto, con desafortunada claridad, cómo la falta de acceso igualitario a bienes básicos y elementales para la vida, y la imposibilidad de ejercer efectivamente los derechos exhiben la crisis de derechos humanos que Chile enfrenta en razón de esta pandemia y que ya se había develado, con mucha fuerza, en la crisis social del 18 de octubre.

Pero esta crisis de derechos humanos afecta de manera distinta a niños, niñas y adolescentes y eso no ha sido entendido ni abordado de manera efectiva por quienes conducen el país. Este es un grupo especialmente vulnerable de nuestra población y el Estado tiene el deber de proteger a sus integrantes de una manera reforzada, algo que, además de no haber sido visible en las acciones gubernamentales, tampoco ha sido informado a ellos y ellas de manera adaptada a su capacidad de comprensión. Los niños, niñas y adolescentes desconocen, en muchos casos, las acciones dirigidas a resolver la situación que enfrenta el país y no han recibido, desde las autoridades, ninguna medida exclusiva que les permita comprender que ellas y ellos son tan dignos e iguales como las personas adultas y que, además, se les asegurará el derecho a participar, considerando su desarrollo evolutivo, con igualdad de oportunidades para que esta crisis no les afecte de manera tan brutal.

Hasta hoy, lo único que ha dado cierta visibilidad a los niños, niñas y adolescentes ha sido la discusión en torno a su situación escolar y el debate sobre el retorno a clases, como si los niños, niñas y adolescentes solo tuvieran desarrollo posible en el ámbito educacional, como si su vida girara en torno a los aspectos relacionados con el aprendizaje formal que se desarrolla en los establecimientos educacionales. Aquella miope visión hace que, en la multiplicidad de ámbitos donde ellas y ellos se desenvuelven, lisa y llanamente no sean visibilizados por el Estado como sujetos de atención y, consecuentemente, como sujetos de protección.

Como Defensoría de la Niñez de Chile, convencidos de la necesidad de que la relación entre el Estado y niños, niñas y adolescentes cambie, y de que esta crisis tan profunda permita "reconstruir" dicha relación de manera virtuosa, solicitamos al gobierno la generación de una mesa de trabajo permanente, transversal e intersectorial que, incluyendo representación participativa e incidente de los propios niños, niñas y adolescentes, permita que todas las autoridades que tienen el deber de ejercer acciones destinadas a prevenir vulneraciones a sus derechos y satisfacer debidamente su protección, diseñen e implementen políticas y acciones públicas con enfoque de derechos humanos que atiendan, de manera específica, a evitar el impacto negativo que esta situación país tiene y tendrá sobre ellas y ellos. Aquella petición no fue atendida, en una manifestación más de que los niños, niñas y adolescentes no son lo primero, que respecto de ellas y ellos no existen prioridades reales, sino que meramente discursivas, y que, incluso en las horas más críticas que enfrenta el país, su afectación no es vista como una realidad que requiere un abordaje prioritario. 
Dicha visión del rol del Estado en relación con niños, niñas y adolescentes no es inocua, pues no solo involucra una mirada del rol estatal que hace primar la lógica del "subsidio" o del "asistencialismo", la que ha predominado por años y pretende mantenerse, sino que devela la grave incapacidad de dar cumplimiento a aquel mandato básico y central que el propio Estado se atribuyó al determinar su obligación relacionada con que "está al servicio de la persona humana y su finalidad es promover el bien común, para lo cual debe contribuir a crear las condiciones sociales que permitan a todos y a cada uno de los integrantes de la comunidad nacional su mayor realización espiritual y material posible", lo que solo puede cumplirse si quienes deben dirigir la actuación estatal entienden qué es lo que viven las personas y qué es lo que necesitan, específicamente qué es lo que viven los niños, niñas y adolescentes y qué necesitan, pues sin ese conocimiento e información no es ni será posible que sus acciones, subsidiarias o no, resulten oportunas, eficientes y efectivas.

Día a día nos enfrentamos a la violencia que representa la inequidad y falta de dignidad en la que viven miles de chilenos y chilenas, la que se agudiza aún más cuando ataca, de manera directa y permanente, a quienes debiéramos proteger de manera prioritaria, a quienes debiéramos alejar de cualquier vulneración de sus derechos. Es difícil que dicha violencia institucional deje de existir respecto de niños, niñas y adolescentes si, incluso en una crisis tan grave como la que enfrentamos, siguen sin ser vistos, sin ser escuchados y sin ser atendidos en igualdad.

Legítimamente, quienes trabajan a diario en la protección de niños, niñas y adolescentes nos hacemos muchas preguntas: ¿qué acciones estatales se han dirigido, de manera específica, a su cuidado, atención, trato y protección? ¿Cómo se ha priorizado a los niños, niñas y adolescentes que se encuentran en situación de pobreza multidimensional y por ingresos? ¿Cuántas de las medidas económicas anunciadas e implementadas por el Ejecutivo han impactado, de manera efectiva, en los niños, niñas y adolescentes que viven en Chile? ¿Cuántas de las medidas económicas han priorizado su entrega a quienes ejercen labores de cuidado y protección de niños, niñas y adolescentes? ¿Cómo se ha abordado la protección de aquellos niños, niñas y adolescentes migrantes que, encontrándose en situación de irregularidad, no pueden acceder al Registro Social de Hogares y, por tanto, a las entregas gubernamentales de alimento y dinero para sobrevivir? ¿Qué acciones de prevención se han llevado a cabo para evitar que la violencia y maltrato contra niños, niñas y adolescentes se produzca mientras se mantiene la situación de confinamiento? ¿Cuántos de los padres, madres o adultos cuidadores de niños, niñas y adolescentes han recibido formación, capacitación y entrega de herramientas suficientes para mantener la crianza en esta situación de crisis sin recurrir a la violencia o el maltrato? ¿Cuántas acciones específicas para generar intervenciones efectivas en el ámbito de la salud 
mental de niños, niñas y adolescentes se han ejecutado desde la administración del Estado, con el fin de evitar que estos hechos afecten significativamente su desarrollo armonioso e integral? La verdad es que la falta de acción favorece la generación de muchas más preguntas, las que hoy no encuentran respuestas de parte de las autoridades o solo hallan algunas llenas de generalidades en las que niños, niñas y adolescentes siguen siendo vistos como meros apéndices de los adultos con quienes se relacionan o de las familias a las que pertenecen.

Unicef ha sostenido que:

Si no se toman medidas urgentes, esta crisis de la salud amenaza con convertirse en una crisis de los derechos de los niños. Los trastornos que ha provocado en la sociedad tienen una profunda repercusión sobre los niños: sobre su seguridad, su bienestar y su futuro. La cooperación multilateral es la única forma de lograr que millones de niñas y niños (incluidos los que han debido abandonar sus hogares a causa de un conflicto, los que padecen una discapacidad y las niñas que están expuestas a diversos peligros) permanezcan sanos, protegidos y sigan recibiendo una educación ${ }^{6}$.

Con la finalidad de evitar que esta crisis se produzca, Unicef releva seis acciones concretas que los Estados debieran ejecutar. Primero, garantizar que todos los niños estén sanosy bien alimentados; segundo, llegar a los niños vulnerables para proporcionarles agua, saneamiento e higiene; tercero, que se facilite el aprendizaje de los niños; cuarto, que se ayude a las familias a cubrir sus necesidades y cuidar a sus hijos; quinto, que se proteja a los niños de la violencia, la explotación y el abuso; y sexto, que se proteja a los niños refugiados, migrantes y a los afectados por un conflicto.

Si analizamos las acciones que el Estado chileno ha realizado en las materias señaladas por estas seis recomendaciones, cabe preguntarse si al no visibilizar a los niños, niñas y adolescentes como sujetos de derecho y víctimas de esta crisis, estas se han podido ejecutar y si, de haberse ejecutado, han puesto o no en el centro de ellas a los niños, niñas y adolescentes y sus necesidades. Con la finalidad de ilustrar de forma ejemplar la situación actual, explicitaré algunos hechos concretos que evidencian la incapacidad del Estado chileno de cumplir estas acciones.

La Junta Nacional de Auxilio Escolar y Becas puso a disposición, a través de la entrega de una caja con víveres, los alimentos que se entregaban en los establecimientos educacionales a cada niño, niña o adolescente registrado en sus

6. Unicef, Plan de Acción Covid-19, en línea en https://www.unicef.org/es/historias/protegerlos-ninos-mas-vulnerables-de-efectos-coronavirus-covid-19. 
programas. Esta distribución, que no estuvo exenta de críticas y reclamos, permitió, en un inicio y de alguna manera hasta ahora, abordar las necesidades de alimento que niños, niñas y adolescentes presentan. Sin perjuicio de aquello, estas últimas semanas hemos sido testigos, en las calles de algunas comunas de nuestro país, de protestas de personas que aseguran no tener acceso a la alimentación, hecho que impacta significativamente en la posibilidad de que niños, niñas y adolescentes estén sanos y bien alimentados. Dichas manifestaciones son coherentes con que, hasta hace dos días, se había entregado solo el 5 por ciento de las cajas de alimentos que el gobierno había comprometido llevar en ayuda de las familias chilenas, dejando abierta la interrogante de cómo están resolviendo su situación alimentaria los niños, niñas y adolescentes del país.

Si consideramos los porcentajes de pobreza por ingresos y a ellos sumamos las prohibiciones de circulación y funcionamiento comercial, medidas destinadas a evitar la propagación de la enfermedad, resulta evidente que las posibilidades de las personas a cargo del cuidado de niños, niñas y adolescentes de tener recursos suficientes para asegurar su alimentación se reducen, y aquello debe implicar acciones decididas del Estado para favorecer, en términos prioritarios, la atención de quienes deberán proveer alimentación a niños, niñas y adolescentes, con el objetivo de asegurar su situación de salud y su desarrollo integral. En esta línea, acciones económicas y de entrega de alimentación debieran considerar, como prioridad, a quienes tienen bajo su responsabilidad el cuidado y protección de este grupo de la población.

Además, y en lo que dice relación con el abordaje sanitario, no solo se ha considerado, de manera bastante ofensiva en un inicio, a los niños, niñas y adolescentes como meros "vectores" de contagio del Covid-19, sino que ahora, cuando ya se ha asumido que también contraen la enfermedad y que, por tanto, requieren ser atendidos e incluso en algunos casos han debido ser hospitalizados, nos encontramos con la inexistencia de protocolos de actuación que les permitan estar cuidados y acompañados, en sus procesos de hospitalización, por un adulto significativo, situación que resulta contraria a su propia condición de niños, niñas y adolescentes y que demanda del Estado el diseño de acciones que, asegurando la debida prevención del contagio, no les exponga a la grave afectación que puede implicar, en términos de su salud mental y de su desarrollo, la obligación de permanecer solos en un recinto asistencial. Este hecho es particularmente grave si consideramos que ni siquiera se han visualizado, dentro de este grupo, las necesidades de acompañamiento que presentan los niños, niñas y adolescentes en situación de discapacidad, las de quienes presentan trastorno de espectro autista u otras condiciones que demandan, necesariamente, de intervenciones médicas especiales que no pueden depender de la sola voluntad de quien atiende. 
Además de lo planteado, hay que recordar que la salud no solo se relaciona con el bienestar físico, sino que involucra la necesidad de contemplar los cuidados necesarios de la salud mental. En estos meses, las atenciones de salud mental de niños, niñas y adolescentes bajo el cuidado del Estado se han visto suspendidas; no existe ningún plan de intervención en este ámbito que se esté desarrollando de manera explícita con participación directa de representantes de los propios niños, niñas y adolescentes, lo que ha profundizado seriamente las posibilidades de que la dimensión emocional de esta crisis sanitaria provoque consecuencias inmediatas y a mediano y largo plazo que luego no seamos capaces de abordar con rigurosidad.

Respecto de la acción de "llegar a los niños vulnerables para proporcionarles agua, saneamiento e higiene", valga la pena mencionar que en Chile, en el año 2020, existen niños, niñas y adolescentes que no tienen acceso al agua, hecho que evidentemente no les permite desarrollarse de manera armoniosa e integral y afecta significativamente su derecho a la vida y a la integridad física y psíquica. Así, y solo a modo ejemplar, en la provincia de Petorca reciben escasos 20 litros de agua para consumo personal diario, en circunstancias de que lo que la Organización Mundial de la Salud establece como cantidad mínima para vivir son 100 litros de consumo diario personal ${ }^{7}$. Dicha flagrante vulneración a los derechos humanos de niños, niñas y adolescentes no ha sido ni atendida ni resuelta por las autoridades sanitarias que, de manera persistente, ofensiva y violenta para estos niños, niñas y adolescentes, refuerzan a diario que la mejor prevención para evitar el contagio del Covid-19 es el lavado de manos frecuente, dejando "correr" el agua por 30 segundos cada dos horas ${ }^{8}$. Esto podría haber sido abordado de manera inmediata por las autoridades sanitarias en razón de la grave situación que representa la pandemia, pero no ha ocurrido y, por el contrario, ellas defienden, en instancias judiciales en las que nos encontramos debatiendo, que con 50 litros es suficiente para que ellas y ellos vivan, desatendiendo de manera evidente el deber de tener, como consideración primordial, el interés superior de niños, niñas y adolescentes a acceder a este elemento vital y a poder estar sanos.

Además de los aspectos precedentes, esta crisis ha dejado de manifiesto las brechas existentes entre los niños, niñas y adolescentes dependiendo de su origen, condición social y lugar de residencia, así como acceso a Internet y a equipos computacionales que les permitan desarrollar el aprendizaje a distancia. Aquellas brechas solo vienen a profundizar otras que la educación chilena no ha sido capaz

7. Organización Mundial de la Salud, en línea en https://www.who.int/water_sanitation_health/ diseases/wsh0302/es/

8. Ministerio de Salud, Chile, Autocuidado Covid-19, en línea en https://www.minsal.cl/nuevocoronavirus-2019-ncov/material-de-descarga/. 
de abordar y que no se originan con la crisis sanitaria, sino que con la incapacidad del Estado de Chile de asegurar educación de calidad en condiciones de igualdad a todos los niños, niñas y adolescentes sin discriminación alguna. Quienes tienen situaciones o condiciones de discapacidad, quienes se encuentran viviendo en la calle, quienes se educan en la ruralidad, quienes están bajo el cuidado del Estado en recintos de protección o privativos de libertad, quienes pertenecen a pueblos indígenas, entre otros, han visto desde hace mucho tiempo dificultado el ejercicio efectivo de su derecho a la educación, lo que solo se ha profundizado en este contexto. No debemos olvidar que la educación se concibe como un derecho humano, pero para que sea efectivamente un derecho y no una mera expectativa para algunos, debe cumplir con el resguardo de principios como la no discriminación, la igualdad de oportunidades y de trato y el acceso universal ${ }^{9}$, situaciones que para un grupo importante de niños, niñas y adolescentes en Chile no son reales.

La educación, para ser concebida como derecho humano, debe entenderse como algo más que la transmisión de contenidos y, de acuerdo con el Comité de Derechos Económicos, Sociales y Culturales, en todas sus formas y en todos los niveles debe tener cuatro características interrelacionadas: la disponibilidad, que implica el deber de existencia de instituciones y programas de enseñanza en cantidad suficiente en el Estado Parte; la accesibilidad, que a su vez involucra tres dimensiones (la no discriminación, es decir, que sea accesible para todos, especialmente para los grupos vulnerables de hecho y de derecho; la accesibilidad material, que implica que debe ser asequible en su localización geográfica como tecnológica; y la accesibilidad económica, que señala que debe estar al alcance de todos); la aceptabilidad, que exige que en la forma y en el fondo los programas de estudio y métodos pedagógicos sean pertinentes, adecuados culturalmente y de buena calidad; y la adaptabilidad, que demanda que la educación sea lo suficientemente flexible para adaptarse a las necesidades de las sociedades en transformación y responder de manera adecuada a las y los alumnos en contextos sociales y culturales diversos.

Poder cumplir con esta educación, entendida como un derecho humano de todas y todos los niños, niñas y adolescentes que viven en Chile, involucra la necesidad de que el Estado adopte medidas urgentes y ejecute acciones que permitan priorizar o adecuar el currículo, identificando contenidos y metodologías, para que exista factibilidad de que los y las estudiantes puedan alcanzar el aprendizaje durante el nuevo escenario escolar, con el fin de permitir un pasaje lo más fluido posible a la educación presencial formal; que se adecue la jornada escolar, permitiendo el

9. Unesco, Derecho a la educación, en línea en https://es.unesco.org/themes/derecho-aeducacion/principios-fundamentales. 
equilibrio necesario entre las horas que los NNA le dedican al estudio durante el periodo de distanciamiento social, de modo de favorecer la continuidad del aprendizaje sin generar un impacto negativo y un estrés adicional al que ya involucra el confinamiento para los NNA; incorporar nuevos contenidos de aprendizaje especialmente diseñados para atender las necesidades de los NNA en contexto de crisis, donde resultan prioritarios los énfasis en aquellos contenidos y competencias relacionadas con el vínculo personal, los aprendizajes para la vida y la entrega de habilidades para el desarrollo de esta; y establecer instancias de evaluación constante de las medidas adoptadas en atención a la crisis sanitaria, incorporando las recomendaciones de organismos técnicos y, sobre todo, de representantes de todos los estamentos de las comunidades educativas, con prioritaria participación de los propios niños, niñas y adolescentes.

Vistos estos elementos, como algunos ejemplos de la falta de consideración primordial de los derechos de niños, niñas y adolescentes, creo que la posibilidad de abordar la situación de la infancia y la adolescencia en este contexto de crisis, con la transversalidad pretendida y en un marco de acción intersectorial, es lo único que permitiría no solo atender, con eficacia y de manera oportuna, la crisis y la contingencia que hoy estos enfrentan sino que, además, proyectar, en virtud de lo que ellas y ellos están viviendo, la mejor forma de conducir acciones en los ámbitos en los que los NNA deben desenvolverse, asegurándoles un trato debido en el futuro inmediato y en el largo plazo, donde su condición de sujetos de derecho se transforme en algo concreto, real, y deje de ser una mera declaración verbal.

Aquella relación que la sociedad construye y desarrolla con los niños, niñas y adolescentes está, muchas veces, mediada por la forma en que el propio Estado, a través de sus diversos órganos, se relaciona con ellas y ellos, exhibiendo comportamientos que luego se replican en el comportamiento ciudadano, lo que afecta significativamente las posibilidades de desarrollo, en el máximo de sus potencialidades, de este grupo de la población. Si el Estado no da espacios para oír y participar a niños, niñas y adolescentes en aquellas instancias donde se resuelven acciones o medidas que les afectarán, lo que hace no solo es vedar un espacio de reconocimiento de ellas y ellos como un o una otro/a válido/a que tiene derecho a un trato de igualdad y dignidad, en tanto sujeto de derecho, sino que, además, incumple de manera manifiesta la obligación jurídica que adquirió al ratificar la Convención sobre los Derechos del Niño, esto es, asegurarles espacios de escucha y tener en cuenta su opinión.

Espero y confío que la crisis social del 18 de octubre de 2019 y esta crisis sanitaria de la pandemia Covid-19, devenida en crisis de derechos humanos, obligue a quienes tenemos un profundo y real compromiso con la democracia en nuestro país a mirar con detención el rol del Estado, a repensar la forma en la que este 
ha construido el desarrollo del país y a costa de la vida de cuántos y cuántas ese desarrollo se ha producido; a mirar con atención y rigor los llamados que los niños, niñas y adolescentes hacen, desde hace mucho tiempo, para que les veamos, les escuchemos y les demos espacios; a asumir nuestras responsabilidades como adultos y observar autocríticamente cómo, al impedirles participar, hemos impactado en aquellas búsquedas de espacios de atención por vías que les exponen a situaciones riesgosas; a entender que necesitamos un marco jurídico suficientemente capaz de asegurar el ejercicio efectivo de los derechos humanos; a comprometernos con la no regresividad en este ámbito y que nuestra intervención propenda a que aquellas victorias que se creía haber obtenido con las declaraciones y tratados internacionales de derechos humanos dejen de ser solo victorias documentales y declarativas y trasunten en experiencias de vida de quienes, con sus luchas, las hicieron posibles, impactando de manera efectiva, concreta y real en el día a día de cada habitante de nuestro país. Solo así podremos asegurar que Chile es un país que no se limita a firmar los tratados internacionales, sino que los promueve, respeta y cumple, asegurando a todos sus habitantes aquella dignidad e igualdad que declara concretar y que pone al servicio de la persona humana, no esperando que sea esta quien, de manera servil, favorezca el desarrollo de las instituciones que deben brindarle protección y cuidado. 